\title{
Effect of Notches on Magnetic Induction Variation for Magnetostrictive Clad Plate
}

\author{
Zhenjun Yang, Hiroki Kurita, Hiroki Takeuchi, Kenya Nakajima, and Fumio Narita ${ }^{*}$ \\ Department of Materials Processing, Graduate School of Engineering, Tohoku University, Sendai, Japan
}

(Received September 29, 2018; accepted January 16, 2019)

Keywords: electromagnetic mechanics, simulation and testing, iron-cobalt alloys, notch, energy harvesting

This study focuses on the notch effect on $\mathrm{FeCo} / \mathrm{Ni}$ clad plate cantilevers. We investigated the response of the cantilevers to an external load and the energy conversion performance. The three-point bending test well demonstrated that the presence of notches can broaden the variation of magnetic induction with respect to an external load. Furthermore, this variation is proportional to the load. The maximum output voltage of the notched $\mathrm{FeCo} / \mathrm{Ni}$ clad plate cantilever is 2.4 times that of the cantilever without a notch. The following vibration energy harvesting experiment also revealed that the output voltage of the former was far greater than that of the $\mathrm{FeCo} / \mathrm{Ni}$ clad plate cantilever without a notch by a factor of 2 . The result of finite element analysis (FEA) indicated that the improvement of the energy harvesting performance was the result of localized stress concentration around the notches in response to an external load. In addition, the $\mathrm{FeCo} / \mathrm{Ni}$ clad plate cantilever showed a superposed enhancement of the energy harvesting performance compared with a single FeCo plate cantilever as a result of the different properties of the FeCo and Ni alloys.

\section{Introduction}

Magnetostrictive materials have been attracting considerable attention not only in the fields of sensors and actuators but also as promising energy harvesters to solve the energy problem. Over the past decades, the properties of magnetostrictive materials have undergone numerous significant developments stemming from the exploration of novel materials and the improvement of processing techniques, ${ }^{(1-3)}$ especially in strength, magnetostriction, and thermal stability, for example. The feasible applications of magnetostrictive materials are therefore broadened. Thus, the applications of magnetostrictive materials in harvesting ambient energy have been gaining increased attention. ${ }^{(4,5)}$

To harvest ambient energy such as vibration, several magnetostrictive materials have been widely explored for use as high-performance harvesters for energy conversion. ${ }^{(6-8)}$ Terfenol-D, owing to its excellent magnetostriction, is a possible candidate. However, owing to its natural drawbacks such as extreme brittleness, further applications have been obviously constrained

*Corresponding author: e-mail: narita@material.tohoku.ac.jp

https://doi.org/10.18494/SAM.2019.2146 
in this field. Hence, many researchers have fabricated a series of polymer-matrix composites at the expense of lower magnetostriction to improve the mechanical properties. Nevertheless, the final harvesting performance exhibited a limited improvement. ${ }^{(9,10)}$ On the other hand, considering the mechanical properties, a series of metallic alloys, such as galfenol and ironcobalt alloys, have been considered as feasible substitutes. In this case, a number of experiments on evaluating the relevant harvesting performance have been reported. ${ }^{(11-13)}$ In particular, an $\mathrm{FeCo} / \mathrm{Ni}$ magnetostrictive cantilever showing a high performance in harvesting vibration energy has been proposed. ${ }^{(14)}$ However, most works have focused on the improvement of material properties through some subsequent processing techniques, considering the relatively low magnetostriction for most metallic alloys. ${ }^{(15,16)}$ Therefore, the processing procedure has some restriction requirements regarding the equipment employed, and in this case, increasing cost and sophisticated processing are inevitable.

A common consensus that the structural design actually has a significant effect on harvesting performance is becoming increasingly important. On the basis of this theory, various designs ${ }^{(17,18)}$ for magnetostrictive composites in different structures, such as 0-3 and 1-3 models, were prepared. Although the harvesting performance generally exhibits an obvious improvement, the aforementioned structural designs are always used to consider the combination of different constituents while there is often a lack of designs for a single constituent. However, considering the harvesting features of magnetostrictive harvesters, an external load is essential for exciting the magnetic induction variation of a magnetized magnetostrictive material to generate electric power in accordance with Faraday's law. Namely, there are alternative feasible designs for every single constituent to amplify loading through an appropriate structural design. As a consequence, the final harvesting performance is further enhanced. Our previous work ${ }^{(19)}$ revealed that the presence of a notch for a single magnetostrictive material can increase the magnetic induction variation through localized stress concentration.

In this study, we aim to evaluate the effect of notches on magnetic induction variation in different magnetostrictive clad plates. The $\mathrm{FeCo} / \mathrm{Ni}$ clad plate was employed to measure the variation of magnetic induction under different loadings. Combined with finite element analysis (FEA), our work is aimed at providing insights into the factors affecting energy conversion in the presence of notches. We also determine the feasibility of magnetostrictive composites with irregular shapes in further improving the harvesting performance.

\section{Experiment}

We considered a clad plate with notches to serve as a vibration energy harvester in this study. Cold-rolled FeCo and Ni were joined by thermal diffusion bonding. During this process, the residual stresses of the $\mathrm{FeCo}$ and Ni layers were 104.5 and $44.5 \mathrm{MPa}$, respectively. ${ }^{(20)}$ After that, the bonded plate was cut into several cantilevers with the same dimensions of $70 \times 5 \times 1 \mathrm{~mm}^{3}$. The harvesting performance characteristics of two groups, namely, non-notch and notch groups, were compared. Here, two notches were introduced at the center along both long sides for the notch group, as shown in Fig. 1. A rectangular Cartesian coordinate system, O-xyz, was used and the origin of the coordinate system coincides with the center of the plate 


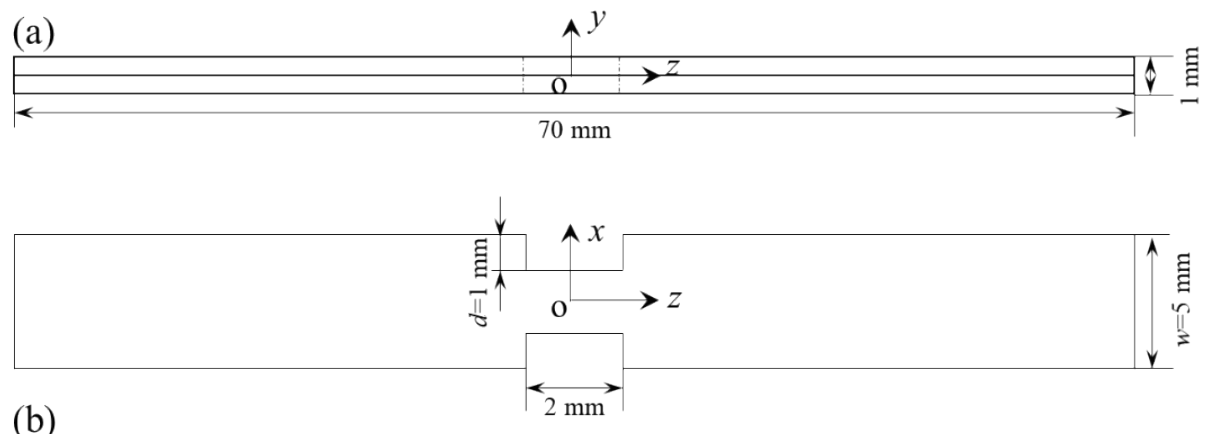

(b)

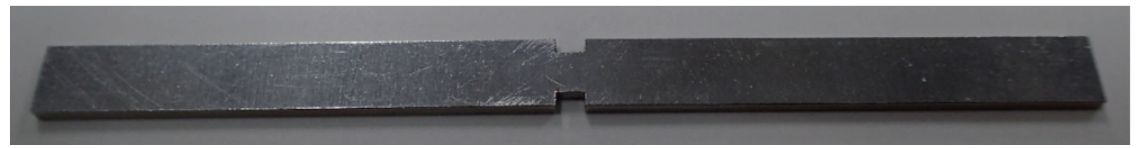

Fig. 1. (a) Schematics and (b) photograph of the $\mathrm{FeCo} / \mathrm{Ni}$ clad plate.

[see Fig. 1(a)]. The depth and width of the notch for the practical experiment were 1 and 2 $\mathrm{mm}$, respectively. Three-point bending [Fig. 2 (a)] was employed to evaluate the effect of the notches on magnetic induction variation in the $\mathrm{FeCo} / \mathrm{Ni}$ clad plate. A bias magnetic field of $370 \mathrm{mT}$ positioned at the two terminals was applied to magnetize the plate in the three-point bending experiment. The loading force $(P)$ ranged from 0 to $25 \mathrm{~N}$ for both groups. A Tesla meter (M) was positioned at a point near $x=w / 2-d, y=0$, and $z=0$ to measure the real-time variation of magnetic induction. In addition, the vibration energy harvesting performance was evaluated taking into account the effect of the notches around a practical circumstance, as shown in Fig. 2(b). This process was carried out at various frequencies in the range of 50 to $200 \mathrm{~Hz}$ to elucidate the resonance frequency for each group and then compare the maximum output voltages of the two groups. A function generator was used to apply a sinusoidal signal to drive the vibration shaker (m030/MA1; IMV Corporation, Japan). The induced voltage of the clad plate cantilever was measured with a data logger connected to the coil setup outside the clad plate cantilever. The coil was wound with $2.5 \times 10^{4}$ turns and had a resistance of $13.5 \mathrm{k} \Omega$. The vibration was conducted at an acceleration of $10 \mathrm{~g}$. An outside loading resistance with the same resistance of $13.5 \mathrm{k} \Omega$ was connected to the coil turns. There is no bias magnetic field in the vibration experiment. On the other hand, FEA was used to examine the details with respect to the energy conversion mechanism through the distributions of stress and magnetic induction. In particular, the specific harvesting power density, as well as the specific distributions of the stress and magnetic induction on a cross-sectional plane, was also examined. Here, we must emphasize that the simulation parameters are almost the same as those in the practical experiments.

\section{Formula and Theoretical Model}

To analyze the details of stress concentration and magnetic induction variation in the FeCo clad plate cantilever in response to an external load, FEA was employed to facilitate the understanding of the conversion mechanism. The Cartesian coordinates $x_{i}(i=1,2,3)$ were 

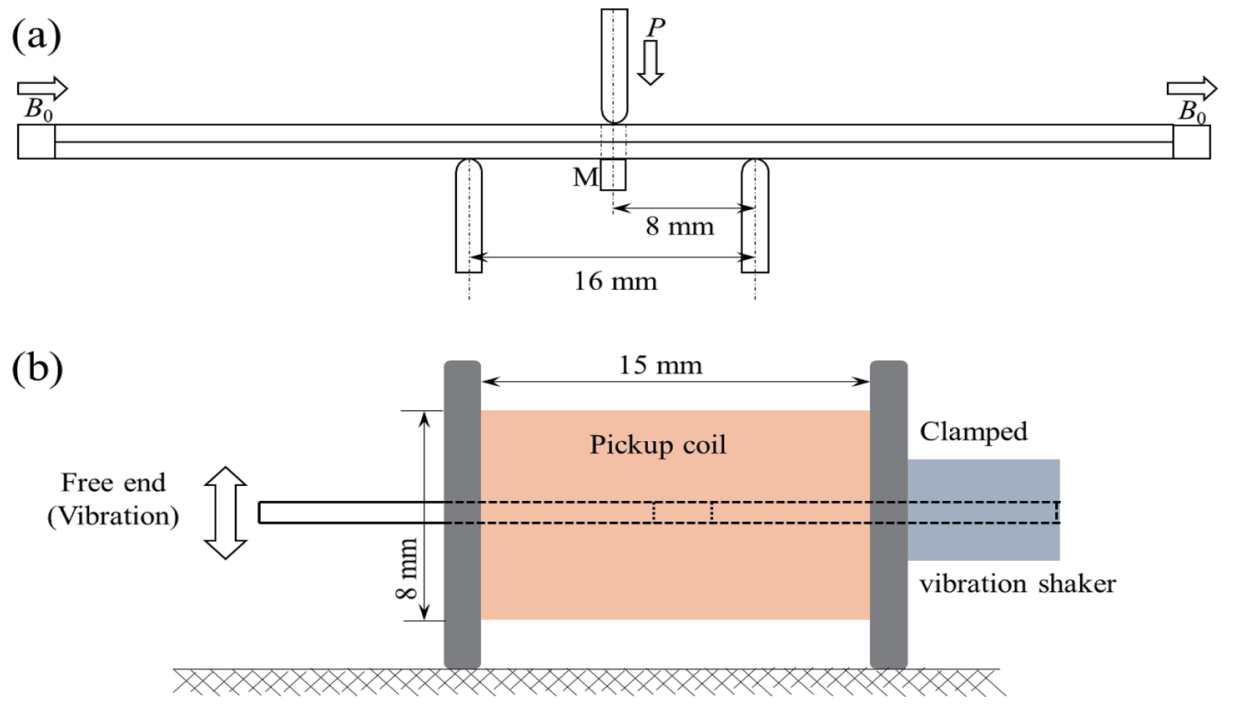

Fig. 2. (Color online) Schematics of (a) three-point bending test and (b) vibration test.

used and the constitutive equations of magnetostrictive materials are given by

$$
\begin{gathered}
\varepsilon_{i j}=s_{i j k l}^{H} \sigma_{k l}+d_{k i j}^{\prime} H_{k}, \\
B_{i}=d_{k i j}^{\prime} \sigma_{k l}+\mu_{i k} H_{k},
\end{gathered}
$$

where $\sigma_{i j}, \varepsilon_{i j}, H_{i}$, and $B_{i}$ denote the components of the stress tensor, strain tensor, magnetic field intensity vector, and magnetic induction vector, respectively. In addition, $s_{i j k l}^{H}, d_{k i j}^{\prime}$, and $\mu_{i k}$ are the constant magnetic field elastic compliance, magnetoelastic constant, and magnetic permeability that meet the following symmetry relations:

$$
s_{i j k l}^{H}=s_{j i k l}^{H}=s_{i j l k}^{H} s_{k l i j}^{H}, d_{k i j}^{\prime}=d_{k j i}^{\prime}, \mu_{i j}=\mu_{j i} .
$$

The constitutive Eqs. (1) and (2) can be respectively expanded as

$$
\left\{\begin{array}{c}
\varepsilon_{11} \\
\varepsilon_{22} \\
\varepsilon_{33} \\
2 \varepsilon_{23} \\
2 \varepsilon_{31} \\
2 \varepsilon_{12}
\end{array}\right\}=\left[\begin{array}{cccccc}
s_{11}^{H} & s_{12}^{H} & s_{13}^{H} & 0 & 0 & 0 \\
s_{12}^{H} & s_{11}^{H} & s_{13}^{H} & 0 & 0 & 0 \\
s_{13}^{H} & s_{13}^{H} & s_{33}^{H} & 0 & 0 & 0 \\
0 & 0 & 0 & s_{44}^{H} & 0 & 0 \\
0 & 0 & 0 & 0 & s_{44}^{H} & 0 \\
0 & 0 & 0 & 0 & 0 & s_{66}^{H}
\end{array}\right]\left\{\begin{array}{c}
\sigma_{11} \\
\sigma_{22} \\
\sigma_{33} \\
\sigma_{23} \\
\sigma_{31} \\
\sigma_{12}
\end{array}\right\}+\left[\begin{array}{ccc}
0 & 0 & d_{31}^{\prime} \\
0 & 0 & d_{31}^{\prime} \\
0 & 0 & d_{33}^{\prime} \\
0 & d_{15}^{\prime} & 0 \\
d_{15}^{\prime} & 0 & 0 \\
0 & 0 & 0
\end{array}\right]\left\{\begin{array}{l}
H_{1} \\
H_{2} \\
H_{3}
\end{array}\right\},
$$




$$
\left\{\begin{array}{l}
B_{1} \\
B_{2} \\
B_{3}
\end{array}\right\}=\left[\begin{array}{cccccc}
0 & 0 & 0 & 0 & d_{15}^{\prime} & 0 \\
0 & 0 & 0 & d_{15}^{\prime} & 0 & 0 \\
d_{31}^{\prime} & d_{31}^{\prime} & d_{33}^{\prime} & 0 & 0 & 0
\end{array}\right]\left\{\begin{array}{l}
\sigma_{11} \\
\sigma_{22} \\
\sigma_{33} \\
\sigma_{23} \\
\sigma_{31} \\
\sigma_{12}
\end{array}\right\}+\left[\begin{array}{ccc}
\mu_{11} & 0 & 0 \\
0 & \mu_{11} & 0 \\
0 & 0 & \mu_{33}
\end{array}\right]\left\{\begin{array}{l}
H_{1} \\
H_{2} \\
H_{3}
\end{array}\right\},
$$

where

$$
\begin{gathered}
\sigma_{23}=\sigma_{32}, \sigma_{31}=\sigma_{13}, \sigma_{12}=\sigma_{21}, \\
\varepsilon_{23}=\varepsilon_{32}, \varepsilon_{13}=\varepsilon_{31}, \varepsilon_{12}=\varepsilon_{21}, \\
s_{11}^{H}=s_{1111}^{H}=s_{2222}^{H}, s_{12}^{H}=s_{1122}^{H}, s_{13}^{H}=s_{1133}^{H}=s_{2233}^{H}, s_{33}^{H}=s_{3333}^{H}, \\
s_{44}^{H}=4 s_{2323}^{H}=4 s_{3131}^{H}, s_{66}^{H}=4 s_{1212}^{H}=2\left(s_{11}^{H}-s_{12}^{H}\right), \\
d_{15}^{\prime}=2 d_{131}^{\prime}=2 d_{223}^{\prime}, d_{31}^{\prime}=d_{311}^{\prime}=d_{322}^{\prime}, d_{33}^{\prime}=d_{333}^{\prime} .
\end{gathered}
$$

A three-dimension FEA model was created in this work to calculate the distributions of the stress and magnetic induction. Here, $z=x_{3}$ was chosen as the easy axis of magnetization. In this case, there is a bias magnetic field along the length direction (easy axis), and the longitudinal (33) magnetostrictive deformation is dominant. Thus, the constants $d_{15}^{\prime}, d_{31}^{\prime}$, and $d_{33}^{\prime}$ can be given by

$$
\begin{gathered}
d_{15}^{\prime}=d_{15}^{m} \\
d_{31}^{\prime}=d_{31}^{m} \\
d_{33}^{\prime}=d_{33}^{m}+m_{33} H_{3},
\end{gathered}
$$

where $d_{15}^{m}, d_{31}^{m}$, and $d_{33}^{m}$ are piezomagnetic constants, and $m_{33}$ is the second-order magnetoelastic constant. The basic equations for the magnetostrictive materials are mathematically equivalent to those for piezoelectric materials. Thus, the commercial package ANSYS with coupled-field solid, acoustic fluid, and infinite acoustic elements was used in the simulation. Here, the bias magnetic field $B_{0}$ and compressive loading $P$ are along the $z$-direction, that is, the easy axis.

The simulation parameters (e.g., $B_{0}$ and $P$ ) were almost the same as those in the practical experiments. The properties of $\mathrm{FeCo}^{(19,21)}$ and $\mathrm{Ni}$ alloys related to the calculation by FEA are listed in Table 1. The relative permeability $\mathrm{m}_{11}$ was assumed to be zero. In addition, $m_{33}$ was $0.0123 \times 10^{-2} \mathrm{~m}^{2} \mathrm{~A}^{-2}$ for the alloys and the magnetic permeability $\mu_{0}$ of the free space was $1.26 \times 10^{-6} \mathrm{H} \mathrm{m}^{-1}$. On the other hand, an air layer, whose dimensions were assumed to be $75 \times$ $15 \times 25 \mathrm{~mm}^{3}$, was employed in this simulation. Furthermore, an air density of $1.2 \mathrm{~kg} / \mathrm{m}^{3}$ was assumed in calculating the magnetic field distribution in air. During the simulation process, 
FEA is also employed to analyze the harvested energy density through the following equation:

$$
Q=\frac{1}{V}\left(\frac{B_{1} H_{1}}{2}+\frac{B_{2} H_{2}}{2}+\frac{B_{3} H_{3}}{2}\right)
$$

where $V$ is the volume of the notched clad plate cantilever.

\section{Results and Discussion}

Figure 3 shows the variations of magnetic induction of both $\mathrm{FeCo} / \mathrm{Ni}$ clad plates with and without notches under various loads ranging from 0 to $25 \mathrm{~N}$. The results indicate that the variations of magnetic induction of the $\mathrm{FeCo} / \mathrm{Ni}$ plates both with and without notches are proportional to external load. It can also be found that this variation of magnetic induction of the plate with notches significantly increases compared with that of the plate without notches. The maximum variation in the former is approximately 2.4 times that in the plate without notches at a load of $25 \mathrm{~N}$. Furthermore, the difference between these two groups increases with the increasing load. This phenomenon is related to the increase in localized stress concentration as a result of the existence of notches. The practical vibration energy harvesting performance in the range of 30 to $200 \mathrm{~Hz}$ is also shown in Fig. 4. The results indicate that these two $\mathrm{FeCo} / \mathrm{Ni}$

Table 1

Material properties of FeCo and Ni alloys.

\begin{tabular}{|c|c|c|c|c|c|c|c|c|c|c|c|}
\hline \multirow[t]{2}{*}{ Material } & \multicolumn{6}{|c|}{$\begin{array}{l}\text { Elastic compliance } \\
\left(\times 10^{-12} \mathrm{~m}^{2} \mathrm{~N}^{-1}\right)\end{array}$} & \multicolumn{3}{|c|}{$\begin{array}{l}\text { Piezomagnetic constant } \\
\qquad\left(\times 10^{-12} \mathrm{~m}^{2} \mathrm{~A}^{-1}\right)\end{array}$} & \multicolumn{2}{|c|}{$\begin{array}{c}\text { Relative } \\
\text { permeability }\end{array}$} \\
\hline & $s_{11}^{H}$ & $s_{33}^{H}$ & $s_{44}^{H}$ & $s_{66}^{H}$ & $s_{12}^{H}$ & $s_{13}^{H}$ & $d_{31}$ & $d_{33}$ & $d_{15}$ & $\mu_{11}$ & $\mu_{33}$ \\
\hline $\mathrm{FeCo}$ & 5.5 & 5.5 & 14.3 & 14.3 & -1.65 & -1.65 & -60.3 & 125 & 318 & 0 & 30 \\
\hline$\overline{\mathrm{Ni}}$ & 5 & 5 & 13.1 & 13.1 & -1.55 & -1.55 & 35.5 & -73.6 & -187.2 & 0 & 351 \\
\hline
\end{tabular}

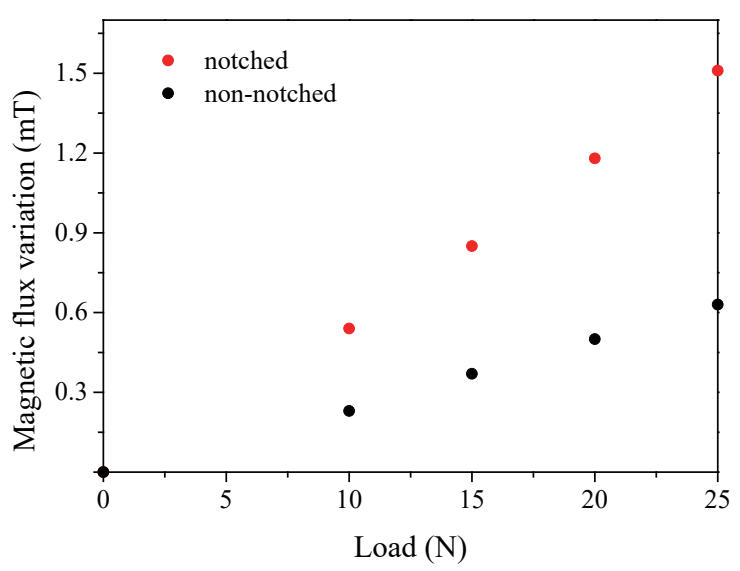

Fig. 3. (Color online) Comparison of the variation of magnetic induction for the $\mathrm{FeCo} / \mathrm{Ni}$ clad plates with and without notches in load range of 0 to $25 \mathrm{~N}$.

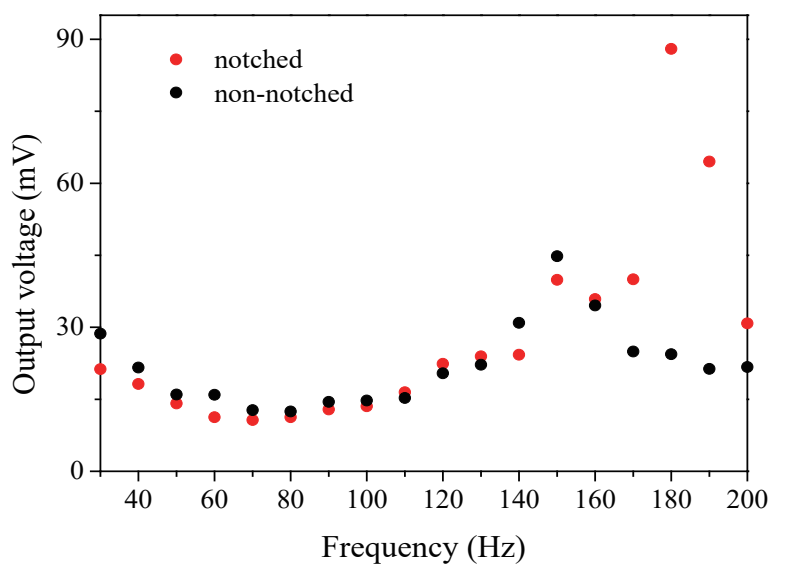

Fig. 4. (Color online) Output voltage for the $\mathrm{FeCo} /$ $\mathrm{Ni}$ clad plate cantilevers with and without notches under various frequencies. 
clad plate cantilevers with and without notches have a similar output performance at a relatively low frequency. With the increase in frequency, the output voltage of the cantilever with notches shows a gradual upward tendency up to a value far greater than that of the cantilever without notches. Here, the resonance frequencies for the $\mathrm{FeCo} / \mathrm{Ni}$ clad plate cantilevers with and without notches are different, i.e., 150 and $185 \mathrm{~Hz}$, respectively. This result was attributed to the existence of notches leading to the change in resonance frequency. Furthermore, it is obvious that the maximum output voltage of the cantilever with notches is far greater than that without notches by a factor of 2 . Here, it must be emphasized that the bias magnetic field is not employed in the vibration experiment. However, according to our former works, the presence of a bias magnetic field improves the output performance substantially. Namely, this notched design can certainly generate more power when a bias magnetic field is employed.

The presence of a notch significantly enhances the output performance of the $\mathrm{FeCo} / \mathrm{Ni}$ clad plate, which has been well demonstrated, as described above. It is also necessary to figure out the mechanism responsible for this notch-related enhancement. Therefore, FEA was employed to examine the relevant details with respect to three-point bending. Here, a single FeCo plate model was used to analyze its difference from the $\mathrm{FeCo} / \mathrm{Ni}$ clad plate with the other parameters of the former (e.g., dimensions and loading) were completely identical to those of the latter. Figure 5 illustrates the distribution of the magnetic induction vector for air (coordinates of $x=$ $0, y=-2.5--0.5, z=-1-+1)$ underneath the notches of the $\mathrm{FeCo} / \mathrm{Ni}$ clad plate under different loads. It is obvious that the magnitude of the magnetic induction vector at a load of $25 \mathrm{~N}$ is greater than that without loading. Thus, the presence of a notch indeed enhances the localized magnetic induction, according to these findings. To clarify the relationship between the load and the magnetic induction flux variation under three-point bending, the stress distribution resulting from the external load around the notch is shown in Fig. 6. Here, the half models of

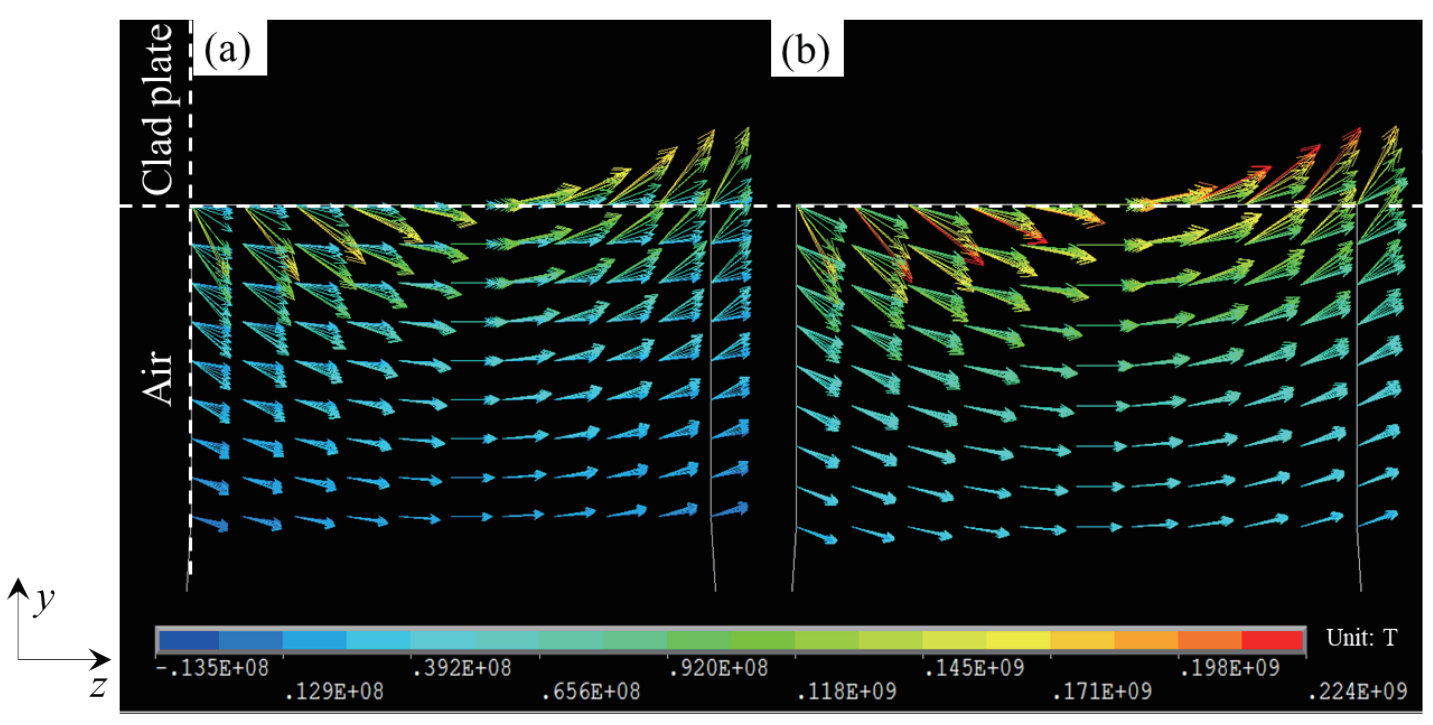

Fig. 5. (Color online) Distribution of magnetic induction vector in the air below the $\mathrm{FeCo} / \mathrm{Ni}$ clad plate with notches at the coordinates of $x=0, y=-2.5--0.5, z=-1-+1$ and with loads of (a) 0 and (b) $25 \mathrm{~N}$. 

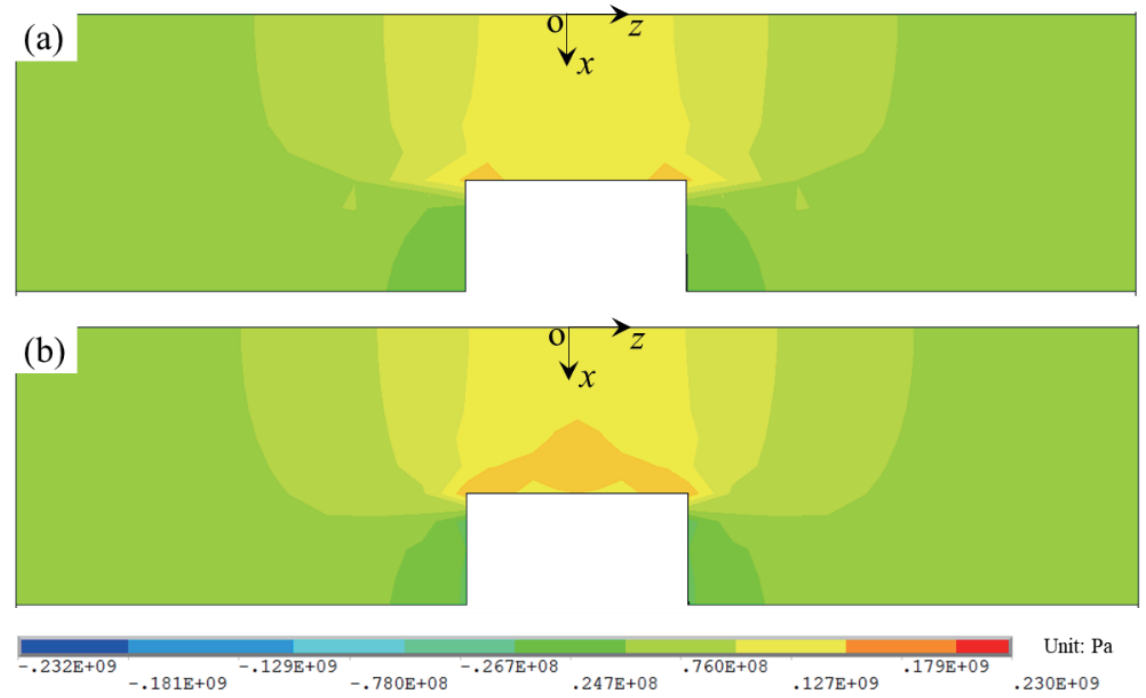

Fig. 6. (Color online) Results of FEA analysis of the distribution of stress under three-point bending for the (a) single FeCo and (b) FeCo/Ni plates.

the single $\mathrm{FeCo}$ and $\mathrm{FeCo} / \mathrm{Ni}$ plates were used to perform FEA. Figure 6(b) clearly shows stress concentration around the notch of the $\mathrm{FeCo} / \mathrm{Ni}$ clad plate. It is interesting that the distributions of stress concentration in the single $\mathrm{FeCo}$ and $\mathrm{FeCo} / \mathrm{Ni}$ plates are distinct in response to the same loading. In particular, the changeable range of the $\mathrm{FeCo} / \mathrm{Ni}$ clad plate was obviously greater than that of the singe FeCo plate. This is attributed to the different properties of FeCo and Ni. In more detail, because of the different properties (e.g., elastic modulus and magnetostriction), the stress transference within single $\mathrm{FeCo}$ and $\mathrm{FeCo} / \mathrm{Ni}$, especially around the $y=0$ plane, is different, then leading to distinct stress concentration. Figure 7 shows the notable effects of elastic compliances on the stress concentration in the $\mathrm{FeCo} / \mathrm{Ni}$ plate at a specific dot $(x=-1.5 \mathrm{~mm}, y=-0.5 \mathrm{~mm}, z=1 \mathrm{~mm})$, as determined by FEA. Here, the double elastic compliances of FeCo and Ni alloys were assumed individually. Furthermore, the stresses resulting from the variation in every elastic compliance were examined. Our findings well demonstrated that the elastic compliance $s_{33}$ significantly affects the stress concentration. On the other hand, combined with the particular magnetostrictive properties of the FeCo and $\mathrm{Ni}$ alloys, the localized stress concentration shows an extra improvement of magnetic induction variation. As a consequence, the final output voltage undergoes further improvement. The details with respect to the superposed enhancement due to the notch for the $\mathrm{FeCo} / \mathrm{Ni}$ clad plate are discussed below.

The FEA of three-point bending of the single $\mathrm{FeCo}$ and $\mathrm{FeCo} / \mathrm{Ni}$ plates with notches was also carried out to evaluate the theoretical energy conversion efficiency. Table 2 indicates that the variations of energy density of the single $\mathrm{FeCo}$ and $\mathrm{FeCo} / \mathrm{Ni}$ plates during the threepoint bending test from 0 to $25 \mathrm{~N}$ are 15.48 and $433.64 \mathrm{~J} / \mathrm{mm}^{3}$, respectively. Here, the energy density of the $\mathrm{FeCo} / \mathrm{Ni}$ clad plate is far greater than that of the single $\mathrm{FeCo}$ plate, a maximum of approximately 29 times. There are two possible reasons for this interesting phenomenon. First, 

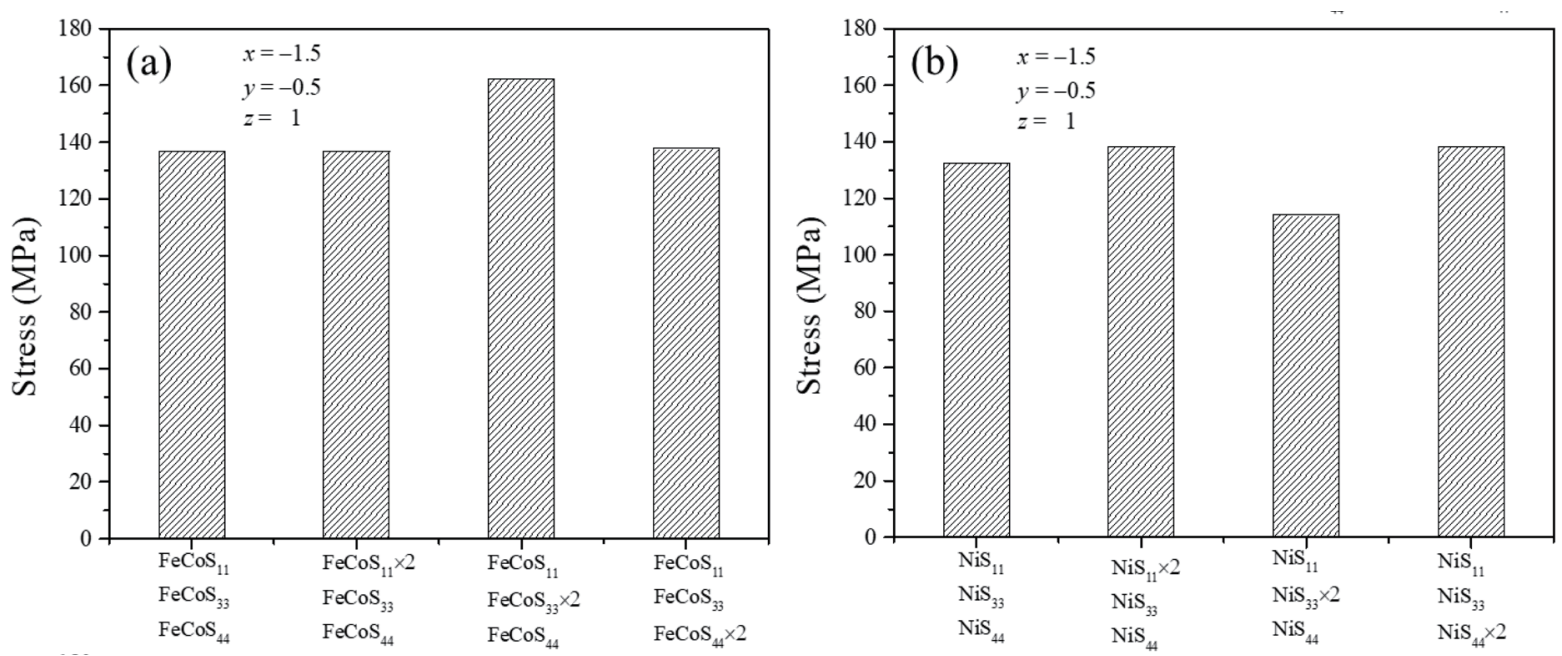

Fig. 7. Variation of stresses as a function of the elastic compliances of (a) FeCo and (b) Ni.

Table 2

Harvested energy density as a function of load.

\begin{tabular}{ccc}
\hline \multirow{2}{*}{ Load $(\mathrm{N})$} & \multicolumn{2}{c}{ Energy density $\left(\mathrm{J} / \mathrm{mm}^{3}\right)$} \\
\cline { 2 - 3 } & $\mathrm{FeCo}$ & $\mathrm{FeCo} / \mathrm{Ni}$ \\
\hline 0 & 0 & 0 \\
25 & 15.48 & 433.64 \\
\hline
\end{tabular}

because of the mechanical properties of the FeCo and Ni alloys, the different elastic moduli for these two materials certainly give rise to different deformations in response to identical external load. As a consequence, the stress concentration around the notch differs, which has been well demonstrated in Fig. 6. Hence, the greater magnitude of stress concentration due to the distinct mechanical properties of these two layers in the $\mathrm{FeCo} / \mathrm{Ni}$ clad plate is responsible for the considerable difference. Secondly, the distinct magnetostrictive properties of these two layers of the $\mathrm{FeCo}$ and $\mathrm{Ni}$ alloys also lead to the significant improvement of energy density in comparison with the single FeCo plate, which has been clearly explained in our previous work. ${ }^{(14)}$ Namely, the FeCo alloy has a positive magnetostriction; in contrast, the Ni alloy is characterized by a negative magnetostriction. During the three-point bending process, the upper and lower layers exhibit inverse responses, that is, compression and tension, respectively. In this case, the FeCo and $\mathrm{Ni}$ alloys can therefore excite the same unidirectional magnetic induction vector along the $z$-direction (i.e., easy axis). On the other hand, the single FeCo plate exhibits the cancellation of the magnetic induction vector between the upper and lower layers, which gives rise to a relatively low energy density. Here, it must be emphasized that the simulation result of three-point bending is far greater than that in the practical vibration energy harvesting experiment. The different experimental parameters are indeed responsible for that. Moreover, the practical volume of the FeCo clad plate cantilever for harvesting energy during vibration is only the part enclosed by the pickup coil. 


\section{Conclusion}

The effect of notches on the variation of the magnetic induction in the $\mathrm{FeCo} / \mathrm{Ni}$ clad plate cantilever was demonstrated in this work. The practical three-point bending experiment revealed that the existence of notches increases the variation of magnetic induction, leading to the generation of a higher output voltage. Through the comparison between the $\mathrm{FeCo} / \mathrm{Ni}$ clad plate cantilevers with and without notches, the relevant variation in the former was found to be far greater than that in the latter by a factor of 2.4. In addition, the following vibration energy harvesting experiment also indicated that the practical harvesting performance of the notched $\mathrm{FeCo} / \mathrm{Ni}$ clad plate cantilever was approximately 2 times that of the non-notched $\mathrm{FeCo} / \mathrm{Ni}$ clad plate cantilever. On the other hand, combined with FEA, the localized stress concentration around the notch in response to an external load was found to improve the harvesting performance. Moreover, the FeCo clad plate cantilever with notches exhibited a superposed enhancement compared with the single FeCo plate cantilever with notches because of the different properties of the $\mathrm{FeCo}$ and Ni alloys. The notchlike irregular design further improved the vibration energy harvesting performance, especially for the magnetostrictive composite.

\section{References}

1 W. Wang, J. Cao, N. Zhang, J. Lin, and W. H. Liao: Energ. Convers. Manage. 132 (2017) 189.

2 M. M. Li, J. H. Li, X. Q. Bao, X. Mu, and X. X. Gao: Mater. Design 135 (2017) 197.

3 J. Li, M. Li, X. Mu, X. Bao, and X. Gao: Mater. Trans. 59 (2018) 679.

4 F. Narita and M. Fox: Adv. Eng. Mater. 20 (2018) 1700743.

5 S. M. Na, J. J. Park, S. Lee, S. Y. Jeong, and A. B. Flatau: Mater. Lett. 213 (2018) 326.

6 Z. X. Deng and M. J. Dapino: Smart Mater. Struct. 24 (2015) 125019.

7 A. Yoffe and D. Shilo: Smart Mater. Struct. 26 (2017) 065007.

8 V. V. Palacheva, A. Emdadi, F. Emeis, I. A. Bobrikov, A. M. Balagurov, S. V. Divinski, G. Wilde, and I. S. Golovin: Acta Mater. 130 (2017) 229.

9 I. M. Nascimento, G. Chesini, J. M. Baptista, C. M. B. Cordeiro, and P. A. S. Jorge: IEEE Sens. J 17 (2017) 6615.

10 Z. X. Deng: J. Appl. Phys. 122 (2017) 043901.

11 F. Narita: Adv. Eng. Mater. 19 (2017) 1600586.

12 M. Ito, K. Kamada, A. Yoshikawa, T. Kawamata, S. Fujieda, S. Suzuki, T. Minamitani, and T. Ueno: J. Alloys Compd. 731 (2018) 898.

13 Y. J. Han, H. Wang, T. L. Zhang, Y. K. He, and C. B. Jiang: Appl. Phys. Lett. 112 (2018) 082402.

14 Z. J. Yang, K. Nakajima, R. Onodera, T. Tayama, D. Chiba, and F. Narita: Appl. Phys. Lett. 112 (2018) 073902.

15 P. Taheri, R. Barua, J. Hsu, M. Zamanpour, Y. Chen, and V. G. Harris: J. Alloys Compd. 661 (2016) 306.

16 U. M. Kannan, S. Kuntz, O. Berg, W. Kittler, H. Basumatary, J. A. Chelvane, C. Surgers, and S. N. Jammalamadaka: Appl. Phys. Lett. 108 (2016) 242408.

17 N. Adelsberg, Y. Weber, A. Yoffe, and D. Shilo: Smart Mater. Struct. 26 (2017) 065013.

18 Z. X. Deng and M. J. Dapino: Smart Mater. Struct. 26 (2017)103001.

19 Z. J. Yang, H. Kurita, H. Takeuchi, K. Katabira, and F. Narita: Adv. Eng. Mater. 21 (2019) 1800811.

20 Z. J. Yang, H. Kurita, R. Onodera, T. Tayama, D. Chiba, and F. Narita: Smart Mater. Struct. 28 (2019) 034001.

21 F. Narita and K. Katabira: Mater. Trans. 58 (2017) 302. 